\title{
Pulmonary Artery Pressure and Variations in Arterial Blood Pressure During the Induction Period of General Anesthesia in Elderly Patients
}

\author{
Yulin Liu ${ }^{1}$, Fei Chen ${ }^{1}$, Weibing Zhao ${ }^{2}$ and Ying Zhu ${ }^{1}$ \\ ${ }^{1}$ Department of Anesthesiology, Chongqing Emergency Medical Center (Chongqing University Central Hospital), Chongqing, China \\ ${ }^{2}$ Department of Anesthesiology, Chongqing Public Health Medical Center, Chongqing, China
}

\begin{abstract}
Objective: To determine the correlation of old age, pulmonary artery pressure (PAP) and mean arterial blood pressure (MABP) during anesthetic induction in elderly patients.

Study Design: Prospective cohort study.

Place and Duration of Study: Central Hospital affiliated to Chongqing Medical University from September 2016 to September 2019.

Methodology: A total of 75 elderly patients with pulmonary hypertension, who underwent surgery under general anesthesia, were inducted. Each 25 patients were allocated into 65-74 years, 75-84 years, and $\geq 85$ years groups. PAP was invasively measured using a right heart floating catheter before induction, while MAP was measured using an invasive radial artery catheter at one minute before and within five minutes after anesthetic induction.

Results: PAP and PAMP, both increased with age $(p<0.001)$. MAP at one minute before induction significantly increased with aging, while MAP measured at 1, 3 and 5 minutes after anesthetic induction, exhibited tendencies of significant decrease with aging $(p<0.05)$. The correlation analysis results revealed that mean MAP during anesthesia induction was significantly associated with PASP before induction ( $r=-0.384$ and -0.532 for Kendall and Spearman correlation analyses; both $p<0.05$ ).

Conclusion: Increased PAP may be involved in the association between with aging and increased risk of hypotension during induction.
\end{abstract}

Key Words: General anesthesia, Hypotension, Pulmonary arterial blood pressure, Old age.

How to cite this article: Liu Y, Chen F, Zhao W, Zhu Y. Pulmonary Artery Pressure and Variations in Arterial Blood Pressure During the Induction Period of General Anesthesia in Elderly Patients. J Coll Physicians Surg Pak 2021; 31(01):8-13.

\section{INTRODUCTION}

Patientsundergoing general anesthesiaarevulnerabletohemodynamic disorders, which mainly include reduced cardiac output and hypotension, particularly during the induction of general anesthesia. ${ }^{1,2}$ In a large cohort study, in which hypotension during induction was defined as a mean arterial blood pressure (MAP) decrease of $>40 \%$ and a MAP of $<70 \mathrm{mmHg}$ or MAP $<$ $60 \mathrm{mmHg}$. The incidence of hypotension during the induction period of general anesthesia could reach as high as $13.3 \%{ }^{3}$ Importantly, patients who develop hypotension during the induction of general anesthesia have been correlated to poor prognosis. $^{4}$

Correspondence to: Dr. Ying Zhu, Department of Anesthe-

siology, Chongqing Emergency Medical Center

(Chongqing University Central Hospital), Chongqing,

China

E-mail: lyl20576226@sina.com

Received: June 08, 2020; Revised: December 21, 2020;

Accepted: December 30, 2020

DOI: https://doi.org/10.29271/jcpsp.2021.01.8
Previous studies have indicated that these patients, when compared with patients who did not develop hypotension during the induction period, had a higher risk for incidences of many serious clinical complications, such as heart failure, acute kidney insufficiency, and cerebral and myocardial ischemia. ${ }^{5}$ Therefore, the identification of patients vulnerable to hypotension during the induction of general anesthesia is of important clinical significance.

It has been suggested that the development of hypotension during the induction of general anesthesia may involve many factors, such as the anesthetics used and the characteristics of patients undergoing anesthesia. ${ }^{6}$ Since almost all medications forgeneral anesthesia havemyocardialsuppressive orvasodilatory efficacies, uncovering the patient characteristics related to increased risk of hypotension during induction would be more important and practical. Accumulating evidence suggests that aging is an important risk factor for the development of hypotension during anesthesia induction. ${ }^{7}$ Conventionally, elderly patients have been considered to have reduced cardiovascular reserve, when compared to younger patients, particularly those with reduced reserve of left ventricular systolic function. ${ }^{8}$ 
Furthermore, blunted baroreflex, as a characteristic of impaired autonomic function common in elderly patients, may also account for the potential causes of perioperative hypotension in these patients.

However, no particular attention has been given to the role of pulmonary artery hypertension (PAH)-related right ventricular insufficiency during the pathogenesis of intraoperative hypotension in elderly patients. In the present study, it was hypothesised that aging is associated with increased pulmonary arterial pressure (PAP), which may cause right ventricular systolic dysfunction and volume insufficiency of the left ventricle; thereby, finally leading to hypotension during the induction periods of general anesthesia.

In order to reflect the above association, the present study aimed to evaluate the association betweenage and PAPparameters in elderly patients undergoing general anesthesia, and explore the potential correlation between the age-related increase in PAP parameters and reduction in MAP during the induction periods of general anesthesia.

\section{METHODOLOGY}

The protocol of the study was registered at Chinese Clinical Trial Registry (http://www.chictr.org.cn. No. ChiCTR1900027673); and was approved by the Ethics Committee of the Central Hospital affiliated to Chongqing Medical University. Consecutive patients were included between September 2016 and September 2019. The criteria to be met were patients who were of $>65$ years at enrollment; attended the hospital to receive surgery under general anesthesia, which mainly included surgeries of the abdomen, such as those for hepatobiliary diseases and urological diseases; diagnosed as pulmonary hypertension with moderate or severe tricuspid regurgitation on transthoracic echocardiograph and underwent invasive measurements of PAP and MAP applied before anesthesia induction. The exclusion criteria were heart failure with left ventricular ejection fraction $<50 \%$, hypertensive cardiomyopathy, arrhythmia with significant tachycardia, or bradycardia that may affect cardiac output and systematic blood pressure pulmonary diseases, including chronic obstructive pulmonary diseases, lung cancer, pulmonary fibrosis, severe asthma, or acute respiratory distress syndrome. Furthermore, patients who required surgeries that may affect pulmonary circulation or arterial blood pressure were also excluded, including patients who required surgeries for thorax diseases and heart diseases.

The included patients were grouped according to their age into group 1 (65-74 years), group 2 (75-84 years) and group 3 ( $\geq 85$ years). Overall, a total of 75 patients fulfilled the inclusion criteria, and 25 patients were allocated to each age group.

All of the included patients underwent invasive measurement of PAP in a standard manner in this centre. Swan-Ganz Catheter made from B Braun Medical Ltd. Briefly, Registration number: 20143035019. Briefly, the procedure was performed with the electrocardiographic monitoring system placed via electrodes on the surface of the body. Under local anesthesia with $1 \%$ lido- caine, the right internal jugular vein of the patient was punctured by an experienced anesthesiologist, and the catheterisation was subsequently performed. Then, a floating catheter was placed through the right internal jugular vein into the right atrium. Judging by the changes in pressure curves on the monitors, the floating catheter was placed forward to the right ventricle through the tricuspid valve. After inflation, the floating catheter went forward with the blood flow into the pulmonary artery, with typical pressure curves shown on the screen of the monitor. Subsequently, the parameters of PAP, including pulmonary arterial mean pressure (PAMP) and pulmonary arterial systolic pressure (PASP), were recorded for further analysis.

All of the included patients also underwent invasive measurement of MAP during the induction of general anesthesia via the invasive radial artery catheterisation method. Disposable blood pressure sensor made from B Braun Medical Ltd. Briefly, Registration number: 20170003. Allen's test was performed for each included patient. Subsequently, under local anesthesia with $1 \%$ lidocaine, the left radial artery was punctured by an experienced anesthesiologist, and an invasive radial artery catheter was placed and connected to a pressure transducer to record the changes in arterial blood pressure during the induction period of anesthesia. Then, patients were required to maintain deep breaths with an oxygen mask for five minutes, and the induction of the anesthesia was performed with combined regimens of sedative, analgesic and muscle relaxant, including intravenous midazolam $(0.05 \mathrm{mg} / \mathrm{kg})$, sulfentanyl $(0.6 \mathrm{ug} / \mathrm{kg})$, and cisatracurium $(0.2 \mathrm{mg} / \mathrm{kg})$. In general, sodium acetate ringer's injection was performed at $7 \sim 8 \mathrm{rml} / \mathrm{kg} \cdot \mathrm{h}$ without abnormalities. After five minutes of bag-mask ventilation of $100 \%$ oxygen, endotracheal catheterisation was performed, and the general anesthesia machine was connected. The general anesthesia was maintained with sevoflurane (1.5\%). Using the pressure transducer connected to the radial artery catheter, MAP at one minute before the induction (T1), and at one minute (T2), three minutes (T3), and five minutes (T4) after induction were measured for each patient.

The sample size was obtained from the preliminary experiment and software of Power and Sample Size application. SPSS 19.0 software was used for data management and statistical analysis. Qualitative data were expressed as frequency and percentage, and were compared with using Chi-square test. Continuous variables were presented as mean \pm standard deviation (SD). Multivariate logistic regression analysis was performed to screen the risk factors for hypotension in the induction period. The normality of distribution of continuous variables was tested by histogram normal curve. Non-normal distributed continuous variables were transformed to normal distributed variables by data transformation. Continuous variables with normal distribution were compared using one-way analysis of the variance (ANOVA) with Dunnet's t-test post-hoc analysis. Spearman and Kendall correlation analyses were performed to evaluate the association between PASP before anesthesia and mean MABP after anesthesia induction (mean values of MAP at T2, T3 and T4). Ap-value of $<0.05$ was considered statistically significant. 
Table I: Baseline characteristics of the included patients according to age group.

\begin{tabular}{|c|c|c|c|c|}
\hline Age group (years) & Height (m) & Body weight $(\mathrm{Kg})$ & BMI $\left(\mathrm{Kg} / \mathrm{m}^{2}\right)$ & $\begin{array}{c}\text { Gender } \\
\text { (Male / Femal) }\end{array}$ \\
\hline 65 - 74 (1group) & $1.65 \pm 0.1$ & $65.6 \pm 7.6$ & $24.0 \pm 2.6$ & $14(56 \%) / 11(44 \%)$ \\
\hline 75 - 8 (2group) & $1.63 \pm 0.1$ & $64.9 \pm 9.5$ & $24.4 \pm 2.6$ & $13(52 \%) / 12(48 \%)$ \\
\hline$\geq 85$ (3group) & $1.59 \pm 0.1$ & $55.0 \pm 8.8$ & $21.8 \pm 3.0$ & $10(40 \%) / 15(60 \%)$ \\
\hline$P$-value & $P=0.050$ & P॰0.001 & $P=0.002$ & $P=0.500$ \\
\hline
\end{tabular}

Table II: Screening risk factors for hypotension in the induction period.

\begin{tabular}{|c|c|c|c|c|c|}
\hline Factor & B values & SE & Wals & p-values & $\operatorname{Exp}(B)$ \\
\hline Age (years) & 0.160 & 0.072 & 4.959 & 0.026 & 1.174 \\
\hline Height (m) & 37.803 & 22.875 & 2.731 & 0.098 & 2.616 \\
\hline Bodyweight (Kg) & -0.456 & 0.289 & 2.486 & 0.115 & 0.634 \\
\hline BMI $\left(\mathrm{Kg} / \mathrm{m}^{2}\right)$ & 1.205 & 0.743 & 2.627 & 0.105 & 3.336 \\
\hline Gender & 0.782 & 1.267 & 0.381 & 0.537 & 2.186 \\
\hline PASP $(\mathrm{mmHg})$ & 0.212 & 0.104 & 4.210 & 0.040 & 1.237 \\
\hline
\end{tabular}

\begin{tabular}{|c|c|c|c|c|c|c|}
\hline $\begin{array}{l}\text { Age group } \\
\text { (year) }\end{array}$ & $\begin{array}{c}\text { MAP at T1 } \\
(\mathrm{mmHg})\end{array}$ & $\begin{array}{c}\text { MAP at T2 } \\
(\mathrm{mmHg})\end{array}$ & $\begin{array}{l}\text { MAP at T3 } \\
(\mathrm{mmHg})\end{array}$ & $\begin{array}{c}\text { MAP at T4 } \\
(\mathrm{mmHg})\end{array}$ & $\begin{array}{c}\text { PASP } \\
(\mathrm{mmHg})\end{array}$ & $\begin{array}{c}\text { PAMP } \\
(\mathrm{mmHg})\end{array}$ \\
\hline $65-74$ & $93.0 \pm 9.4$ & $84.2 \pm 6.6$ & $81.6 \pm 12.5$ & $83.6 \pm 13.2$ & $22.7 \pm 3.5$ & $15.4 \pm 1.6$ \\
\hline $75-84$ & $102.8 \pm 6.0$ & $74.4 \pm 16.1$ & $68.6 \pm 11.0$ & $61.1 \pm 7.3$ & $27.6 \pm 4.0$ & $19.2 \pm 2.7$ \\
\hline$\geq 85$ & $104.3 \pm 9.6$ & $72.1 \pm 5.0$ & $61.8 \pm 11.1$ & $53.2 \pm 9.2$ & $31.3 \pm 3.1$ & $21.2 \pm 1.8$ \\
\hline$P$-value & $<0.001$ & $<0.001$ & $<0.001$ & $<0.001$ & $<0.001$ & $<0.001$ \\
\hline
\end{tabular}

\section{RESULTS}

A total of 75 patients were included in the present study; whereas, 25 patients were allocated for each age group. The baseline characteristics, including height and gender, were not significantly different among the three age groups of different age (Table I). However, body weight of group 3, compared to group $1(p<0.001)$, and group $2(p=0.001)$, were significantly different.

Logistic regression analysis was performed to screen the risk factors for hypotension in the induction period. The results of multivariate logistic regression analysis (direct entry method) of the above seven related factors showed that PASP and age were independent risk factors of hypotension during induction period $(p=0.026$ and 0.040 , OR $>1$ (Table II).

These results reveal that MAP at one minute before induction significantly increased with aging. However, MAP measured at 1, 3 and 5 minutes after anesthetic induction exhibited tendencies to decrease with aging, which appeared to be most remarkable at five minutes after induction. Furthermore, these results highlight the significant correlation between the reduction in MAP during the induction period of general anesthesia and aging in elderly patients.

The results of the one-way ANOVA analysis indicated that among patients in the three different age groups, the differences in levels of PASP and PAMP were statistically signifi- cant $(p<0.001)$. Furthermore, the post-hoc analysis revealed that PASP and PAMP, both increased with the increase in age in these groups. PASP and PAMP exhibited a tendency to increase with aging before induction (Table III).

The correlation analysis revealed that mean MAP during anesthesia induction was significantly associated with PASP before induction $(r=-0.384$ and -0.532 for Kendall and Spearman correlation analyses, respectively; both $p<0.001$, Figure 1).

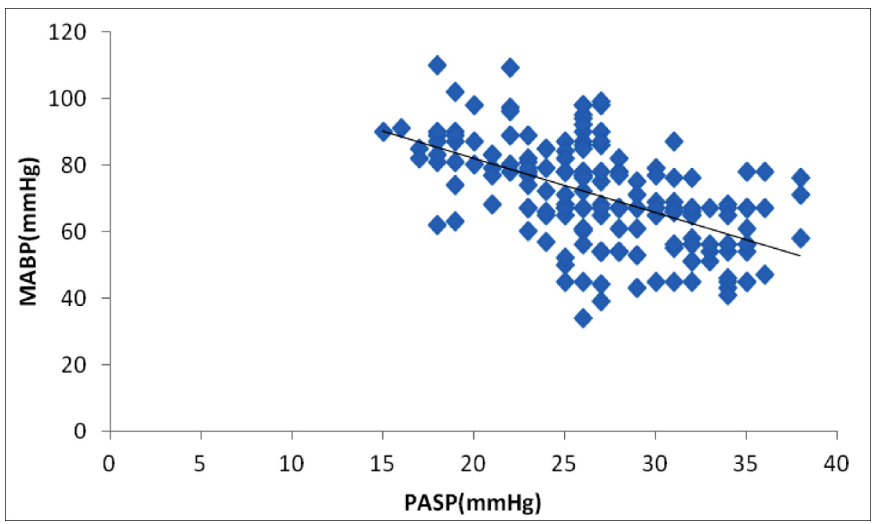

Figure 1: The correlation between PASP before anesthesia induction and mean MAP during induction.

\section{DISCUSSION}

In the present study, all elderly patients, who received general anesthesia, it was found that the parameters of PAP, including PAMP and PASP, increased with the aging of 
patients. Furthermore, it was found that baseline MAP also increased with the aging of patients. However, MAP during the induction periods of general anesthesia became lower with the aging of these included patients.

These results indicate that the reduction in blood pressure during the induction periods of general anesthesia was more remarkable in old patients, suggesting that aging is an important risk factor for the development of hypotension during the induction periods of general anesthesia. Furthermore, aging was correlated with increased PAP in the present study, which highlights that increased PAP may be involved in the association between aging and increased risk of hypotension during induction. In addition, it was found that high pulmonary artery pressure and advanced age were independent risk factors for severe hypotension after induction of general anesthesia. For elderly patients with high pulmonary artery pressure, total anesthetic injection further inhibits the contractile function of the right and left hearts, aggravates pulmonary artery congestion, and affects the pumping function of the heart, resulting in a decrease in peripheral blood pressure and even intractable hypotension.

From the previous studies, the physiological changes of PAP parameters with aging, particularly those with invasive right heart catheterisation-based measurements, remain undetermined. The present study expanded the above findings by showing that PAP parameters may affect elderly patients without significant clinical status, and that PASP and PAMP levels exhibited a tendency to increase with the age of patients in each group, indicating the physiological increase of PAP with aging. The potential mechanisms underlying the physiological change in PAP with aging may be as follows. First, aging leads to reduced compliance of the left ventricle, which may subsequently increase the resistance of pulmonary circulation and increase PAP. ${ }^{9-11}$ Second, aging-related pathophysiological factors, such as hypoxia, and activated inflammatory and oxidative stress-related stimuli, may lead to the remodelling of pulmonary capillaries and arteries, finally causing increased pressure in these arteries. Furthermore, the aging-related decrease in the cross-sectional area of pulmonary capillary beds may also contribute to the elevated pressure in these pulmonary arteries. ${ }^{12-14}$ Taken together, these findings indicate that in the elderly patients undergoing general anesthesia, aging is correlated to elevated PAP.

Although aging has been recognised as an independent risk factor of hypotension during anesthetic induction, 3,15,16 previous studies that investigated the potential mechanisms underlying the aging-related incidence of intraoperative hypotension have mainly focused on changes in left ventricular functions. As previously mentioned, aging is associated with the reduced preservation of systolic function of the left ventricles. $^{8,17,18}$ By applying this accurate measurement method, the present study revealed that although the baseline MAP was higher in patients with advanced age, MAP decreased during the induction periods, suggesting that aging patients are vulnerable to intraoperative hypotension. These findings, together with the findings that PAP increased with aging, enable the conclusion that regardless of the reduced preservation of systolic function in the left ventricles, the right ventricular insufficiency correlated with increased PAP in elderly patients undergoing general anesthesia may also contribute to increased risk of hypotension during anesthetic induction. Since PAH has been confirmed to be an important cause of hemodynamically significant insufficiency of right ventricular function, ${ }^{19,20}$ it is not surprising that the chronic physiological increment of PAP with aging may accordingly lead to chronic pressure overload of the right ventricles. During anesthetic induction, increased PAP and reduced right ventricular function may both contribute to the reduced volume of blood flow, from pulmonary circulation to systematic circulation. To the best of authors' knowledge, few previous studies have investigated the role of $\mathrm{PAH}-$ related right ventricular insufficiency in the aging-related risk of intraoperative hypotension. An extreme example was presented by a recently published case report, which revealed that hypotension during anesthetic induction could be caused by dynamic right ventricular outflow obstruction. ${ }^{21,22}$ Although the increase in PAP was not severe enough to cause right ventricular outflow obstruction in patients in the present study, the potential mechanisms underlying the aging-related intraoperative hypotension may be similar, which at least partially involves the increased PAP-related insufficiency of right ventricular function. It is noteworthy that the volume underload of the left heart is a common cause in these patients; and from a preventive and therapeutic perspective, fluid infusions are important for these patients.

Regardless of the above strengths of the present study, which included the use of invasive methods for the measurement of both PAP and MAP, this present study has limitations that should be considered when interpreting these results. First, the limited sample size of the study. Second, confounding factors may exist underlying the association between aging and increased PAP, and risk of hypotension in the present study. Therefore, the hypothesis that the physiological increase in PAP may expose elderly patients to increased risk of intraoperative hypotension via compromising right ventricular function should be evaluated and tested in future studies.

\section{CONCLUSION}

Increased PAP may be involved in the association between aging and increased risk of hypotension during induction. Furthermore, it should be confirmed whether increased PAP exposes elderly patients to increased risk of hypotension during induction via the impairment of right ventricular function. This may be of preventative and therapeutic significance for intraoperative hypotension in elderly patients. 


\section{FUNDING:}

This work was supported by the Medical Research Project of Chongqing Municipal Health and Family Planning Commission (2017MSXM094).

\section{ETHICAL APPROVAL:}

The protocol of the study had been registered at Chinese Clinical Trial Registry (http://www.chictr.org.cn. No. ChiCTR1900027673), and had been approved by the Ethics Committee of the Central Hospital affiliated to Chongqing Medical University.

\section{PATIENTS' CONSENT:}

As the study was designed retrospectively, data was collected from clinical archives after ethical approval.

\section{CONFLICT OF INTEREST:}

The authors declared no conflict of interest.

\section{AUTHORS' CONTRIBUTION:}

YZ: Substantial contributions to the conception or design of the work; or the acquisition, analysis, or interpretation of data for the work.

FC: Drafting the work or revising it critically for important intellectual content.

WZ: Final approval of the version to be published.

YL: Agreement to be accountable for all aspects of the work in ensuring that questions related to the accuracy or integrity of any part of the work are appropriately investigated and resolved.

\section{REFERENCES}

1. Mets B. Management of hypotension associated with angiotensin-axis blockade and general anesthesia administration. J Cardiothorac Vasc Anesth 2013; 27(1): 156-67. doi: 10.1053/j.jvca.2012.06.014.

2. Juri T, Suehiro K, Tsujimoto S, Kuwata S, Mukai A, Tanaka K, et al. Pre-anesthetic stroke volume variation can predict cardiac output decrease and hypotension during induction of general anesthesia. J Clin Monit Comput 2018; 32(3): 415-22. doi: 10.1007/s10877-017-0038-7.

3. Reich DL, Hossain S, Krol M, Baez B, Patel P, Bernstein A, et al. Predictors of hypotension after induction of general anesthesia. Anesth Analg 2005; 101(3):622-8, table of contents. doi: 10.1213/01.ANE.0000175214.38450.91.

4. Gu WJ, Hou BL, Kwong JSW, Tian X, Qian Y, Cui Y, et al. Association between intraoperative hypotension and 30-day mortality, major adverse cardiac events, and acute kidney injury after non-cardiac surgery: A meta-analysis of cohort studies. Int J Cardiol 2018; 258:68-73. doi: 10.1016/j.ijcard.2018.01.137.

5. Salmasi V, Maheshwari K, Yang D, Mascha EJ, Singh A, Sessler DI, et al. Relationship between intraoperative hypotension, defined by either reduction from baseline or absolute thresholds, and acute kidney and myocardial injury after noncardiac surgery: A retrospective cohort analysis. Anesthesiology 2017; 126(1):47-65. doi: 10.1097/ALN.0000000000001432.
6. Onuigbo MA, Agbasi N. Intraoperative hypotension - a neglected causative factor in hospital-acquired acute kidney injury; a mayo clinic health system experience revisited. J Renal Inj Prev 2015; 4(3):61-7. doi: 10.12861/ jrip.2015.13

7. Luger TJ, Kammerlander C, Luger MF, Kammerlander-Knauer $U$, Gosch M. Mode of anesthesia, mortality and outcome in geriatric patients. Z Gerontol Geriatr 2014; 47(2):110-24. doi: 10.1007/s00391-014-0611-3.

8. Fleg JL, Strait J. Age-associated changes in cardiovascular structure and function: a fertile milieu for future disease. Heart Fail Rev 2012; 17(4-5):545-54. doi: 10.1007/ s10741-011-9270-2.

9. Arbab-Zadeh A, Dijk E, Prasad A, Fu Q, Torres P, Zhang R, et al. Effect of aging and physical activity on left ventricular compliance. Circulation 2004; 110(13):1799-805. doi: 10.1161/01.CIR.0000142863.71285.74.

10. Hur DJ, Sugeng L. Non-invasive Multimodality cardiovascular imaging of the right heart and pulmonary circulation in pulmonary hypertension. Front Cardiovasc Med 2019; 6:24. doi: 10.3389/fcvm.2019.00024.

11. Legris V, Thibault B, Dupuis J, White M, Fortier A, Henri C, et al. Right ventricular function coupling to the pulmonary circulation predicts exercise tolerance in patients with heart failure and reduced ejection fraction. insight from the evaluation of resynchronisation therapy for heart failure (EARTH) trial. J Heart Lung Transplantation 2020; 39(4): S35-S6.

12. Coffman KE, Carlson AR, Miller AD, Johnson BD, Taylor BJ. The effect of aging and cardiorespiratory fitness on the lung diffusing capacity response to exercise in healthy humans. J Appl Physiol (1985) 2017; 122(6):1425-34. doi: 10.1152/japplphysiol.00694.2016.

13. Furukawa K, Yano M, Nishimura M, Nakamura $E$, Watanabe N, Nishino S, et al. Significance of preoperative right ventricular function on mid-term outcomes after surgical ventricular restoration for ischemic cardio-myopathy. Gen Thorac Cardiovasc Surg 2019; 67(11): 925-33. doi: 10.1007/s11748-019-01123-5.

14. Teramoto K, Sengelov M, West E, Santos M, Nadruz W, Skali $\mathrm{H}$, et al. Association of pulmonary hypertension and right ventricular function with exercise capacity in heart failure. ESC Heart Fail 2020; 7(4):1635-44. doi: 10.1002/ehf2. 12717.

15. Shelburne NJ, Parikh KS, Chiswell K, Shaw LK, Sivak J, Arges $\mathrm{K}$, et al. Echocardiographic assessment of right ventricular function and response to therapy in pulmonary arterial hypertension. Am J Cardiol 2019; 124(8):1298-304. doi: 10.1016/j.amjcard.2019.07.026.

16. Ramani G, Chen W, Patel S, Judy J, Ton VK. Noninvasive assessment of right ventricular function in patients with pulmonary arterial hypertension and left ventricular assist device. Curr Cardiol Rep 2019; 21(8):82. doi: 10.1007/ s11886-019-1156-2.

17. $\mathrm{XJ}, \mathrm{ZZ}, \mathrm{CS}$. Evaluation of right ventricular function in pulmonary hypertension with Tei index measured by tissue doppler imaging. Proceeding of Clinical Medicine 2019.

18. Sultan I, Cardounel A, Abdelkarim I, Kilic A, Althouse AD, Sharbaugh MS, et al. Right ventricle to pulmonary artery coupling in patients undergoing transcatheter aortic valve 
implantation. Heart 2019; 105(2):117-21. doi: 10.1136/ heartjnl-2018-313385.

19. Freeman ML, Landolfo C, Safford RE, Keller CA, Heckman $M G$, Burger CD. Noninvasive assessment of right heart function and hemodynamics during exercise in patients with pulmonary arterial hypertension. South Med J 2013; 106(2):141-6. doi: 10.1097/SMJ.0b013e3182805165.

20. Celermajer DS, Marwick T. Echocardiographic and right heart catheterization techniques in patients with pulmonary arterial hypertension. Int J Cardiol 2008; 125(3):294-303. doi: 10.1016/j.ijcard.2007.04.184.

21. Antoniucci ME, Colizzi C, Arlotta G, Calabrese M, Corrado M, Guarneri S, et al. Dynamic right ventricular outflow obstruction: A rare cause of hypotension during anestesia induction. Int J Surg Case Rep 2017; 41:30-2. doi: 10.1016/j.ijscr.2017.08.069.

22. Richter MJ, Fortuni F, Wiegand MA, Dalmer A, Vanderpool R, Ghofrani HA, et al. Association of right atrial conduit phase with right ventricular lusitropic function in pulmonary hypertension. Int J Cardiovasc Imaging 2020; 36(4):633-42. doi: 10.1007/s10554-019-01763-x. 